\title{
Procedimentos para estimar massa de forragem, interceptação luminosa e índice de área foliar em pastos de capim-Tanzânia
}

\author{
Silva, P.H.F. ${ }^{\circledR}$; Anjos, A.N.A. ${ }^{2}$; Viegas, C.R. ${ }^{2}$; Morais, L.F. ${ }^{3}$; Nepomuceno, D.D. ${ }^{2}$ e Carvalho, C.A.B. ${ }^{2}$
}

'Departamento de Zootecnia. Universidade Federal Rural de Pernambuco. Recife. PE. Brasil.

2Departamento de Nutrição Animal e Pastagens/Instituto de Zootecnia. Universidade Federal Rural do Rio de Janeiro. Seropédica. RJ. Brasil. ${ }^{3}$ Departamento de Zootecnia. Universidade Federal do Ceará. Fortaleza. CE. Brasil.

\section{PALAVRAS CHAVE ADICIONAIS}

Pastagem.

Amostragem.

Coeficiente de variação.

Precisão experimental.

\section{RESUMO}

Sob hipótese de ocorrer diferença de precisão entre metodologias de amostragem, objetivou-se avaliar três procedimentos para estimar massa de forragem, altura do pasto, interceptação luminosa e índice de área foliar em pasto homogêneo de capim-Tanzânia, com base em seus coeficientes de variação $(\mathrm{CV})$ e precisões experimentais. Para tanto, foram comparados $\mathrm{CV}$ dos procedimentos de amostragem sistemática, induzida e aleatória. Foram coletadas, em cada procedimento, sete amostras em moldura metálica (unidade de amostragem) de $1,0 \mathrm{~m}^{2}$. A amostragem induzida proporcionou menores $\mathrm{CV}$ acumulados $(2,8 \% ; 16,2 \% ; 3,9 \%$ e $19,06 \%)$ para todas as variáveis. $\mathrm{O}$ procedimento de amostragem induzida permitiv estimar com maior precisão as variáveis avaliadas.

\section{Procedures to estimate forage mass, light interception and leaf area index in Tanzânia grass pastures}

\section{SUMMARY}

\section{ADDITIONAL KEYWORDS}

Pastures.

Sampling.

Variation coefficient.

Accuracy.

INFORMATION

Cronología del artículo.

Recibido/Received: 11.04 .2017

Aceptado/Accepted: 18.05.2018

On-line: 15.07 .2018

Correspondencia a los autores/Contact e-mail:

Pehenrique1709@gmail.com

\section{INTRODUÇÃO}

A massa de forragem é uma ferramenta que visa adequar o manejo eficiente das pastagens, pois permite avaliar parâmetros como taxa de lotação, capacidade de suporte e oferta de forragem (Carvalho et al., 2008 , p. 7). Os melhores métodos de avaliação de massa de forragem são os que representam as condições das pastagens e reduzida mão-de-obra. $\mathrm{O}$ método de amostragem a ser utilizado depende da variabilidade, densidade e composição botânica do pasto, de modo que as características estruturais indicam quais procedimentos e, ou, unidades de amostragem serão mais recomendáveis para se obter resultados mais acurados.

Dentre os diferentes procedimentos existentes, destacam-se a amostragem aleatória, que se baseia na escolha aleatória de pontos a serem amostrados na pastagem, de modo que cada local tenha a mesma chance de ser incluído na amostragem (T'mannetje, 2000, p. 154), o procedimento sistemático que têm como base o estabelecimento de distâncias iguais entre as unidades de amostragem (Ribeiro et al., 2009, p. 1435), indicado para pastos de condições estruturais mais homogêneas, e o procedimento de amostragem "induzida", que constitui-se da escolha de pontos que representem a média de alguma característica do pasto e que tenha correlação positiva com massa de forragem (normalmente a altura média dos pastos).

Entretanto, poucas comparações de procedimentos de amostragem, sobretudo quanto à inclusão da amostragem "induzida" são encontradas na literatura para diferentes espécies forrageiras e, ou, condições 
de manejo. Assim, objetivou-se comparar três procedimentos de amostragem para estimar massa de forragem, altura, interceptação luminosa e índice de área foliar do pasto de capim-Tanzânia com base nos seus coeficientes de variação acumulados e consequentes precisões experimentais.

\section{MATERIAL E MÉTODOS}

O experimento foi conduzido na Fazenda do Instituto de Zootecnia, na Universidade Federal Rural do Rio de Janeiro, Seropédica - RJ (22 $44^{\prime} 38^{\prime \prime}$ S, $43^{\circ} 42^{\prime} 27^{\prime \prime}$ O e 26 metros de altitude). O clima da região é Aw (Köppen) e o solo, Planossolo distrófico. Em 28/11/2015, uma área de $3780 \mathrm{~m}^{2}$ de pasto do capim-Tanzânia (Panicum maximum cv. Tanzânia) manejada sob lotação rotacionada foi escolhida em função de sua maior homogeneidade nas estruturas vertical e horizontal do pasto. Tal área foi subdividida em três parcelas experimentais $\left(1260 \mathrm{~m}^{2}\right.$ cada $)$ e submetidas a três procedimentos de amostragens (sistemática, aleatória e "induzida"), que representaram os tratamentos em experimento sob delineamento inteiramente casualizado.

O número de amostragens (repetições $=7$ ) dentro de cada parcela representou $0,5 \%$ da área, conforme T'Mannetje (2000, p. 156). Somado a isso, em $21 / 11 / 2015$, foram coletadas 30 amostras de forragem, sob procedimento aleatório e em moldura de formato quadrado de $1,0 \mathrm{~m}^{2}$, para estimativa do número mínimo de amostragens necessárias para avaliação da massa de forragem neste pasto de capim-Tanzânia, conforme a metodologia da máxima curvatura do coeficiente de variação de Federer (1955). Os resultados revelaram a necessidade mínima de quatro amostragens para representar a variabilidade deste pasto, já que a partir deste ponto, os coeficientes de variação foram pouco alterados (Santos et al., 2016, p. 2). Assim, o número de repetições $(n=7)$ foi o suficiente para as estimativas realizadas.

Para as avaliações do presente estudo, foi utilizada a mesma moldura da avaliação supracitada $\left(1,0 \mathrm{~m}^{2}\right)$. O material foi cortado (rente ao solo), colhido, acondicionado em sacos plásticos identificados e seco em estufa de circulação forçada de ar a $55^{\circ} \mathrm{C}$ por 72 horas, para determinação das massas de forragem para os três procedimentos.

Em seguida, foram realizadas amostragens para estimativa das alturas, massas de forragem, interceptações luminosas e índices de área foliar do pasto sob os procedimentos de amostragem conforme indicado nas literaturas clássicas de metodologias de avaliação de pastagens (T'mannetje, 2000; Carvalho et al., 2008; Ribeiro et al., 2009).

A amostragem aleatória foi realizada lançando-se, ao acaso, a moldura na área da parcela. Já para a amostragem sistemática, a parcela foi demarcada em três linhas paralelas internas, com distância de 10 metros entre as mesmas. Por fim, a amostragem "induzida" foi realizada com base na escolha de pontos representativos da altura média da parcela $(110 \mathrm{~cm})$, obtida após de 20 medições prévias. Todas as alturas foram obtidas com régua graduada em centímetros. A interceptação luminosa e o índice de área foliar foram estimadas com o aparelho analisador de dossel AccuPAR Linear PAR/LAI ceptometer, Model PAR - 80, à partir de uma leitura acima e três leituras abaixo do dossel forrageiro, sob radiação incidente (sol pleno).

Os resultados foram submetidos a análise de variância pelo software livre $\mathrm{R}$, segundo o modelo $y_{i j}=\mu$ $+t_{i}+e_{i j}$, em que $y_{i j}$ equivale ao valor observado na $j$-ésima amostragem, que recebeu o $i$-ésimo procedimento de amostragem; $\mu$ equivale a média geral; $t_{i}$ equivale ao efeito de procedimento de amostragem e $e_{i j}$ significa o erro experimental. Cada procedimento de amostragem foi considerado um fator e efeito fixo, enquanto que o erro experimental foi considerado efeito aleatório. As médias foram comparadas pelo teste de Tukey, à 5\% de probabilidade do erro. Foram estimados os coeficiente de variação acumulados em cada procedimento para massa de forragem (MF), altura (ALT), interceptação luminosa (IL) e índice de área foliar (IAF) do dossel. As correlações de Pearson entre ALT e MF, e IL e IAF foram obtidas utilizando-se o programa GraphPad Prism versão 7.03 para Windows.

\section{RESULTADOS E DISCUSSÃO}

A área amostrada $\left(7 \mathrm{~m}^{2}\right)$ em cada parcela experimental foi maior que a sugerida por Penati et al. (2005, p. 43) que recomendaram quatro amostras de $1,0 \mathrm{~m}^{2}$ cada para piquetes de $2.000 \mathrm{~m}^{2}$ formados por Panicum maximum e Pennisetum purpureum, utilizando o método da máxima curvatura do coeficiente de variação de Federer (1955). A área amostrada também foi maior do que aquela avaliada por Backes et al. (2018), que retiraram uma amostra de $0,3 \mathrm{~m}^{2}$ em parcela de $10 \mathrm{~m}^{2}$ em pasto de capim marandu (Urochloa brizantha $\mathrm{cv}$. Marandu). Somado a isso, Leal et al. (2017) avaliaram a altura média de pastos de Brachiaria híbrida 'Mulato II' em 3 pontos dentro da moldura metálica de 1,0 $\mathrm{m}^{2}$, assim como realizado no presente experimento. Tais fatos denotam confiabilidade quanto à amostragem das áreas utilizadas.

Os valores de altura (ALT), massa de forragem (MF), interceptação luminosa (IL) e índice de área foliar (IAF), e os respectivos coeficientes de variação acumulados obtidos nos três procedimentos avaliados são apresentados na Tabela I.

Não houve diferença entre alturas para os três métodos de amostragem, contudo foi verificado menor coeficiente de variação acumulado $(\mathrm{CV})$ para amostragem "induzida" em relação as demais. Cutrim Junior et al. (2011 p. 492) obtiveram CV de 8,5\% para estimativa da altura média de pastos avaliada em 30 pontos por piquete $\left(3750 \mathrm{~m}^{2}\right)$, enquanto Rodrigues et al. (2010, p. 590) relataram CV de 7,3 e 12,5\% para as alturas médias de pré-pastejo $(91,8 \mathrm{~cm})$ e pós-pastejo $(37,4 \mathrm{~cm})$ em $500 \mathrm{~m}^{2}$, respectivamente, ambos para capim-Tanzânia. Porém, nos dois trabalhos os autores não descreveram a metodologia de amostragem. Estes resultados superiores ao obtido no procedimento "induzido", o apontam como mais preciso para estimar altura média em pastos homogêneos de capim-Tanzânia. 
Tabela I. Estimativas da altura do pasto, massa de forragem, interceptação luminosa, índice de área foliar do capim-Tanzânia e dos coeficientes de variação acumulados obtidos sob os procedimentos de amostragem sistemática, aleatória e "induzida"(Estimates of sward height forage mass, light interception, leaf area index of Tanzania grass and cumulative variation coefficients obtained under systematic, random and "induced" sampling procedures).

\begin{tabular}{|c|c|c|}
\hline Procedimentos de amostragem & Altura do dossel forrageiro $(\mathrm{cm})$ & CV $(\%)$ \\
\hline Sistemática & $99^{A}$ & 14,9 \\
\hline Aleatória & $101^{\mathrm{A}}$ & 13,4 \\
\hline "Induzida" & $108^{\mathrm{A}}$ & 2,8 \\
\hline \multicolumn{3}{|c|}{ Massa de forragem (Kg ha-1 de massa seca) } \\
\hline Sistemática & $4128^{\mathrm{B}}$ & 32,4 \\
\hline Aleatória & $4328^{\mathrm{B}}$ & 48,7 \\
\hline "Induzida" & $5192^{A}$ & 16,2 \\
\hline \multicolumn{3}{|c|}{ Interceptação luminosa (\%) } \\
\hline Sistemática & $65,9^{B}$ & 25,1 \\
\hline Aleatória & $77,8 A^{B}$ & 12,5 \\
\hline "Induzida" & $88,7^{\mathrm{A}}$ & 3,9 \\
\hline \multicolumn{3}{|c|}{ Índice de área foliar } \\
\hline Sistemática & $2,8^{B}$ & 46,4 \\
\hline Aleatória & $3,3^{\mathrm{AB}}$ & 30,7 \\
\hline “Induzida” & $4,7^{\mathrm{A}}$ & 19,0 \\
\hline
\end{tabular}

Médias de uma mesma variável, seguidas pela mesma letra maiúscula na coluna não diferem entre si pelo teste de Tukey (P>0,05). CV: coeficiente de variação acumulado.

(a)

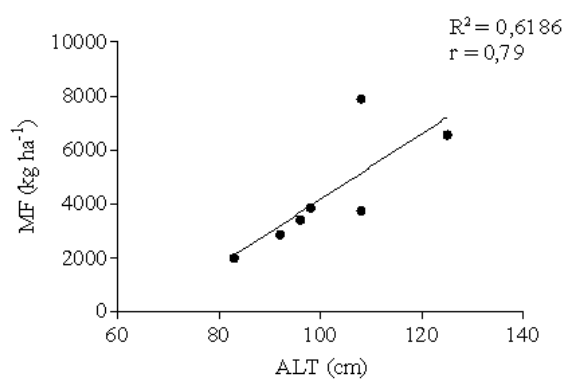

(b)

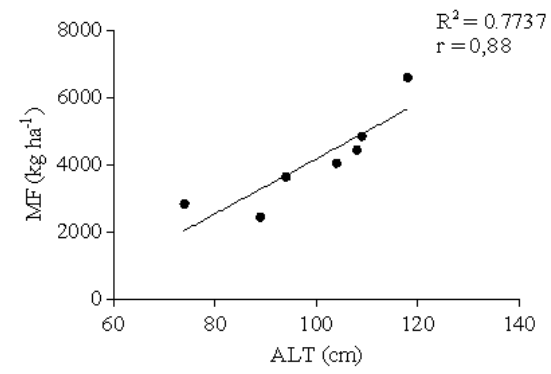

MF: massa de forragem ( $\mathrm{kg} \mathrm{ha}^{-1}$ de massa seca); ALT: altura; (a): procedimento de amostragem aleatória; (b): procedimento de amostragem sistemática; $R^{2}$ : coeficiente de determinação; $r$ coeficiente de correlação de Pearson

Figura 1. Correlação de Pearson entre massa de forragem (MF) estimada em $\mathrm{kg}$ ha-1 de massa seca e altura (ALT) do pasto $(\mathrm{cm})$ para amostragens aleatória (a) e sistemática (b) (Pearson correlation between forage mass (MF) estimated in kg ha-1 of dry mass and sward height $(A L T-c m)$ for random (a) and systematic sampling (b).
Maior valor de massa de forragem $(\mathrm{P}<0,05)$ e menor coeficiente de variação acumulado foram obtidos sob amostragem "induzida" em relação à sistemática ou aleatória. Janusckiewicz et al. (2010, p. 163) reportaram CV de 32,4\% para estimativa da massa de forragem do capim-Tanzânia, utilizando moldura de $1 \mathrm{~m}^{2}$ em piquetes de $55 \mathrm{~m}^{2}$, porém não descreveram o procedimento de amostragem. Contudo, verifica-se que o CV relatado foi semelhante aquele do procedimento sistemático, inferior ao $\mathrm{CV}$ do procedimento aleatório e superior ao CV do procedimento "induzido", o que aponta este último como mais preciso. Também foi verificada correlação alta e positiva entre altura do pasto e massa de forragem (Figura 1) para amostragem aleatória $(\mathrm{r}=0,78 ; \mathrm{p}=0,036)$ e sistemática $(\mathrm{r}=0,88 ; \mathrm{p}=0,009)$, o que indica alto grau de associação entre estas variáveis. Isto denota vantagem para técnicas de manejo com base na altura do pasto, principalmente pela facilidade de aplicação à campo. Quanto ao procedimento de amostragem "induzida", não foi observado efeito significativo $(p=0,99)$, devido à escolha dos sete pontos de coleta com base na altura média, e consequente variação reduzida das alturas e massas de forragem.

Maiores valores de IL e IAF $(\mathrm{P}<0,05)$ foram obtidos sob amostragem "induzida" em relação à sistemática, porém ambos não diferiram do procedimento aleatório. A amostragem "induzida" resultou em menores valores de coeficiente de variação, revelando maior precisão deste procedimento em comparação aos demais. Coêlho et al. (2014, p. 32) avaliaram correlação entre IL e IAF em parcelas experimentais de capimTanzânia, utilizando CI-120 CID BioScience ${ }^{\circledR}$, e observaram correlação positiva $(r=0,69)$. Tal fato também foi observado neste trabalho (Figura 2) para as amos- 
(a)

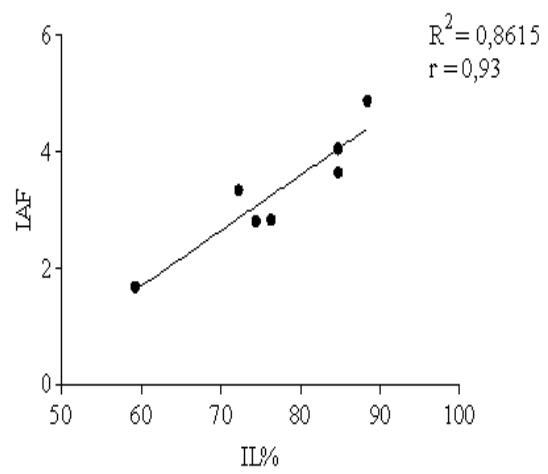

(b)

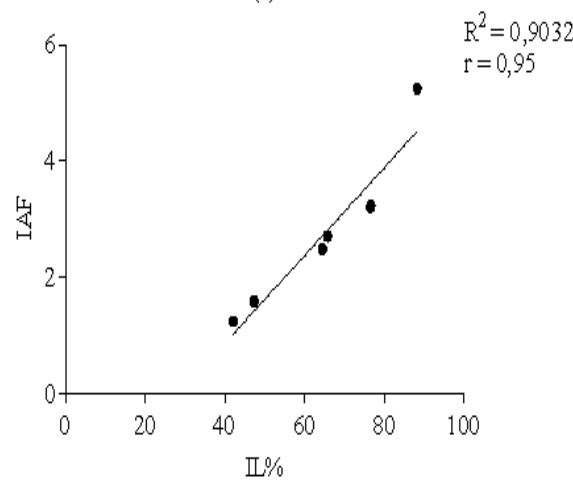

(c)

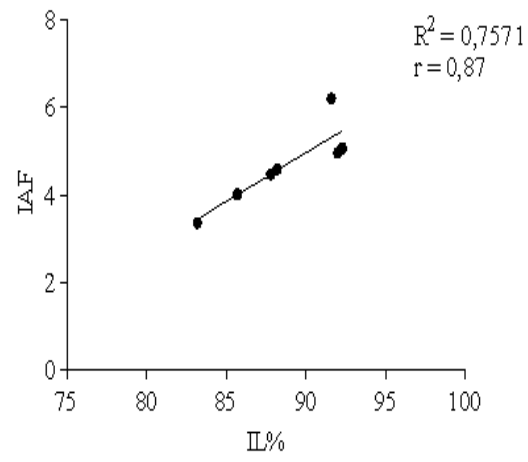

IAF: indice de área foliar; IL: interceptação luminosa (\%); (a) procedimento de amostragem aleatória; (b) procedimento de amostragem sistemática; (c) procedimento de amostragem "induzida"; $R^{2}$. coeficiente de determinação; $r$ : coeficiente de correlação de Pearson.

Figura 2. Correlação de Pearson entre interceptação luminosa (IL\%) e índice de área foliar (IAF) do dossel forrageiro para amostragens aleatória (a), sistemática (b) e "induzida" (c) (Pearson correlation between light interception (IL) and leaf area index (LAI) of forage canopy for random (a), systematic (b) and "induced" sampling (c).

tragens aleatória $(\mathrm{r}=0,93 ; \mathrm{p}=0,002)$, sistemática $(\mathrm{r}=0,95$; $\mathrm{p}=0,010)$ e induzida $(\mathrm{r}=0,76 ; \mathrm{p}=0,011)$.

De acordo com a classificação proposta por Gomes (2009), coeficientes de variação abaixo de 10\% denotam alta precisão experimental, e abaixo de 20\% correspondem à precisão média em experimentos agronômicos à campo. Logo, sob este critério, apenas sob procedimento de amostragem "induzida" obteve-se alta precisão experimental para ALT e IL, e média precisão para MF e IAF.

\section{CONCLUSÃO}

O uso do procedimento de amostragem "induzida" permitiu estimar com maior precisão a massa de forragem, a altura, a interceptação da luminosa e o índice de área foliar em pastos homogêneos de capim-Tanzânia manejados sob lotação rotacionada.

\section{BIBLIOGRAFIA}

Backes, C, Santos, A, Bessa, S, Ribon, A, Teodoro, A, Rodrigues, L, Tomazelo, D, Ferreira, L 2018, 'Taxa de cobertura verde e exportação de macronutrientes pelo capim Marandu em função da aplicação de gesso', Archivos de Zootecnia, vol. 67, no. 2, pp. 234-242.

Carvalho, R, Athayde, A, Valeriano, A, Medeiros, L, Pinto, J 2008, 'Método de determinação da disponibilidade de forragem', Ciência et Praxis, vol. 1, no. 2, pp. 7-10.

Coêlho, J, Júnior, J, Santos, E, Neto, J, Cunha, M, Santos, M, Mello, A, Lira, M 2014, 'Canopy height and its relationship with leaf index and light interception in tropical grasses', Tropical grasslands- forrajes tropicales, vol. 2, no. 1, pp. 31-32.

Cutrim Junior, J, Cândido, M, Valente, B, Carneiro, M, Carneiro, H 2011 , 'Características estruturais do dossel de capim-tanzânia submetido a três frequências de desfolhação e dois resíduos pós-pastejo', Revista Brasileira de Zootecnia, vol. 40, no. 3, pp. 489-497.

Federer, W T 1955, Experimental design, MacMillan, New York, NY.

Gomes, F P 2009, Curso de Estatística Experimental, FEALQ, Piracicaba, SP.

Janusckiewicz, E, Magalhães, M, Ruggieri, A, Reis, R 2010, 'Massa de forragem, composição morfológica e química de Capim-tanzânia sob diferentes dias de descanso e alturas de resíduo pós-pastejo', Bioscience Journal, Uberlândia, vol. 26, no. 2, pp. 161-172.

¡Leal, D, França, A, Oliveira, L, Correa, D, Arnhold, E, Ferreira, R, Bastos, D, Brunes, L 2017, 'Fracionamento de carboidratos e proteínas da Brachiaria híbrida 'Mulato II' sob adubação nitrogenada e regime de cortes', Archivos de Zootecnia, vol. 66, no. 254, pp. 181-188.

Penati, M, Corsi, M, Lima, S, Marta Júnior, G \& Dias, C 2005, 'Número de amostras e relação dimensão: formato da moldura de amostragem para determinação da massa de forragem de gramíneas cespitosas', Revista Brasileira de Zootecnia, vol. 34, no. 1, pp. 36-46.

Prism, Graphpad 2017, Version 7.03, GraphPad Software, Inc., La Jolla, CA.

Santos, A, Anjos, A, Viegas, C, Morais, L, Silva, P, Mello, B, Almeida, J, Brandão, C 2016, Número de amostras para a estimativa da massa de forragem em pastos de capim-Tanzânia' in XXVI Congresso Brasileiro de Zootecnia, Centro de Convenções da UFSM, Santa Maria, pp. 1-3.

Ribeiro, E, Fontes, C, Palieraqui, J, Cóser, A, Martins, C, Silva, R 2009, Influência da irrigação, nas épocas seca e chuvosa, na produção e composição química dos capins napier e mombaça em sistema de lotação intermitente', Revista Brasileira de Zootecnia, vol. 38, no. 8, pp. 1432-1442.

Rodrigues, L, Meirelles, P, Gonçalves, H, Martins, M, Marques, R, Arruda, G 2010, 'Disponibilidade e composição química do capim-łanzânia, pastejado por caprinos', Veterinária e Zootecnia, vol. 14, no. 4, pp. 585-595.

T'mannetje, L 2000, Measuring biomass of grassland vegetation, CABI, Cambridge, MA. 